\title{
Spectroscopic Investigation of Modified Single Wall Carbon Nanotube (SWCNT)
}

\author{
S. Hussain ${ }^{1}$, P. Jha ${ }^{3}$, A. Chouksey ${ }^{3}$, R. $\operatorname{Raman}^{3}$, S. S. Islam ${ }^{1}$, \\ T. Islam ${ }^{2}$, P. K. Choudhary ${ }^{3}$, Harsh $^{3}$ \\ ${ }^{1}$ Department of Applied Sciences and Humanities, Nano Sensor Research Laboratory, \\ Faculty of Engineering \& Technology, Jamia Millia Islamia, India \\ ${ }^{2}$ Department of Electrical Engineering, Faculty of Engineering \& Technology, \\ Jamia Millia Islamia, India \\ ${ }^{3}$ Solid State Physics Laboratory, Delhi, India \\ E-mail: shahirjmi@rediffmail.com \\ Received January 14, 2011; revised March 16, 2011; accepted April 1, 2011
}

\begin{abstract}
We have investigated the effects of chemical treatment on Single Wall Carbon Nanotube (SWCNT) before and after being modified with $\mathrm{HNO}_{3} / \mathrm{H}_{2} \mathrm{SO}_{4}$ by Raman, FTIR and UV-Vis-NIR spectroscopy. The results show successful carboxylation of the CNT sidewalls as observed from FTIR and UV-Vis-NIR spectroscopy. This successful functionalization is achieved in 6-8 hrs of refluxing. We also report changes in the first and second order Raman spectra of SWNTs functionalized with oxygenated groups. During the experiment, we observe some important Raman features: Radial breathing mode (RBM), Tangential mode (G-band), and Disordered mode (D-band); which are affected due to the chemical oxidation of carbon nanotubes. We found that the ratio of D- to the G-band intensity $\left(I_{d} / I_{g}\right)$, increase after functionalization and the RBM mode in acid treated SWCNTs is almost disappeared.
\end{abstract}

Keywords: Single Wall Carbon Nanotubes, Functionalization, Raman Spectroscopy, Ftir and UV-Vis-NIR Spectroscopy

\section{Introduction}

Carbon nanotubes (CNTs) have attracted much attention of researchers worldwide because of their pseudo 1D structure and exceptional mechanical, electrical, and thermal properties [1]. Carbon nanotubes become very useful for many applications such as nanoelectonic devices, chemical sensors, gas sensors, solar cells and so forth.

CNT based sensors, an emerging application area of the nanotubes and have caught the attention of a large number of researchers. Due to the enormously high specific surface area, SWNTs can absorb large amount of gases, which makes them a potential candidate for chemical sensors with very high sensitivity. However, for any practical purpose, apart from sensitivity, selectivity must also be introduced on to the CNT surface; for which the CNT sidewalls/ end caps need to be functionalized with different functional moieties. Functionalization process of CNT decreases van der Waals' forces, leading to de-bundling between the CNT, and aiding binding to other materials [2]. Since carbon nanotubes remain insoluble in common solvents due to the high cohesive energy of more than $0.5 \mathrm{eV} / \mathrm{nm}$ arising from tube - tube van der Waals' attraction, therefore functionalization is also useful in reducing the magnitude of this energy and rendering them soluble [3]. The first step to functionalize the relatively inert CNT surface is to oxidize the CNTs. The various oxidation methods have used for this purpose [4-8]. Liquid phase oxidation is a very simple method to make nanotubes more easily dispersible or soluble in liquids; it is necessary to physically or chemically attach functional groups to their sidewalls without changing significant properties and also opens the closed ends of CNTs [9]. The chemical modification and oxidation of CNTs has been well represented in literature $[10,11]$. During acid oxidation, the carbon-carbon bonded network of the graphitic layers is broken allowing the introduction of oxygen atoms in the form of carboxyl, which has been extensively exploited for further 
chemical functionalization.

It is observed that in the functionalization process, several functional groups such as carboxylic $(-\mathrm{COOH})$, carbonyl $(-\mathrm{CO})$, and hydroxylic $(-\mathrm{OH})$ are formed on the surface of nanotubes, which is typical of carbon materials [12]. It is thought that acid treated nanotubes have carboxylic groups at the tubes ends and possibly, at defects on the sidewalls. H. T. Gomes and co-workers [12] had reported that Carboxylic $(-\mathrm{COOH})$ and carboxylate groups (-COONa) were successfully grafted to the CNT surfaces when modified using $\mathrm{HNO}_{3}, \mathrm{HNO}_{3} / \mathrm{H}_{2} \mathrm{SO}_{4}$, and $\mathrm{HNO}_{3} / \mathrm{Na}_{2} \mathrm{CO}_{3}$.

In this paper, we have used oxidation technique for the modification of SWCNT surface and studied the effect of the chemical treatment on CNT structure using Raman, FTIR and UV-Vis-NIR Spectroscopy. In our studies we have observed that acid treatment duration of only about 6 - 8 hours is sufficient to functionalize the CNTs by introducing $\mathrm{COOH}$ group at the $\mathrm{CNT}$ surface. But to the best of our knowledge most literature cites a reaction duration ranging from 12 - 24 hours. The Raman spectroscopy is a perfect tool for the characterization of carbon nanotube to get important information about their properties without damaging the CNT surfaces [13].

\section{Experimental Detail}

Different sets of experiments were carried out using 100 mg of SWNTs (purity 95\%; length 1 - 2 microns and diameter $<10 \mathrm{~nm}$; manufactured by NTP China) mixed with $3: 1$ ratio of $\mathrm{HNO}_{3}: \mathrm{H}_{2} \mathrm{SO}_{4}$. The CNTs were dispersed in acid mixture by using a multi frequency ultrasonicator (Blackstone NEY). The refluxing technique is used to reflux the solution for better results. For this, the solution was then transferred to a refluxing flask and refluxing was carried out for various time periods using Heidolph LR4011 rotary evaporator. The temperature of refluxing was maintained at $80^{\circ} \mathrm{C}$.

After acid treatment the solution was quenched in ice-cold water, and samples were diluted. Then base neutralization of the acid solution was carried out by adding $0.1 \mathrm{M} \mathrm{NaOH}$. The neutralization point was detected by using a $\mathrm{pH}$ meter (Metler Toledo multi seven).

Thereafter, the CNTs were extracted on a polycarbonate membrane of 0.2-microns pore size using a vacuum filtration assembly. The resulting CNTs were dried in an oven at $80^{\circ} \mathrm{C}$ for $12 \mathrm{hrs}$. Fourier Transform Infrared spectroscopy (FTIR) data was obtained using Biorad FTS 40 spectrometer. Raman data was recorded on a Lab RAM HR 800 Raman spectrometer (Horiba Jobin Yvon,) using Argon $(\mathrm{Ar}+)$ laser at $488 \mathrm{~nm}$ wavelengths. UV-Vis-NIR spectrophotometer data was acquired using Varian Cary 5E spectrophotometer.

\section{Result \& Discussion}

\subsection{Raman Analyses}

There exist many distinct bands in the SWCNT Raman spectrum that originate from different aspects of the nanotube are Radial breathing mode (RBM), Tangential mode (G-band), and Disordered mode (D-band) [13]. Radial breathing modes (RBM) between 100 and 300 $\mathrm{cm}^{-1}$ depends on the tube diameter and this region shows much variation with different samples. The higher frequency tangential displacement $\mathrm{G}$ modes near $\sim 1590$ $\mathrm{cm}^{-1}$ and the second order $\mathrm{G}^{\prime}$ peaks near $2600 \mathrm{~cm}^{-1}$ are sensitive to the charge exchanged between nanotubes and the guest moiety. The shape and the intensity of D-mode at $1290-1320 \mathrm{~cm}^{-1}$ corresponds to the $\mathrm{sp}^{3}-$ hybridized carbon atoms [4], which is correlated with the extent of nanotube sidewall defects and chemical sidewall functionalization [14]. The $\mathrm{D}^{*}$-mode also serves to show the disorder and defects of graphitic walls.

Raman spectra were recorded at room temperature on pristine as well as on acid treated carbon nanotubes using a micro Raman spectrometer equipped with a $488 \mathrm{~nm}$ laser as shown in Figure 1. The strong background in the spectrum observed from the acid treated sample is probably indicative of the fact that the sample surface was rough due to presence of defects during the acid treatment. Chemical oxidation creates defects on the sidewalls of nanotube and attaches some functional group at the defective area of nanotubes. Typical SWCNT Raman features are observed for the RBM- and G-modes at $160-1591 \mathrm{~cm}^{-1}$, respectively. On functionalization there are distinct changes in the Raman spectra

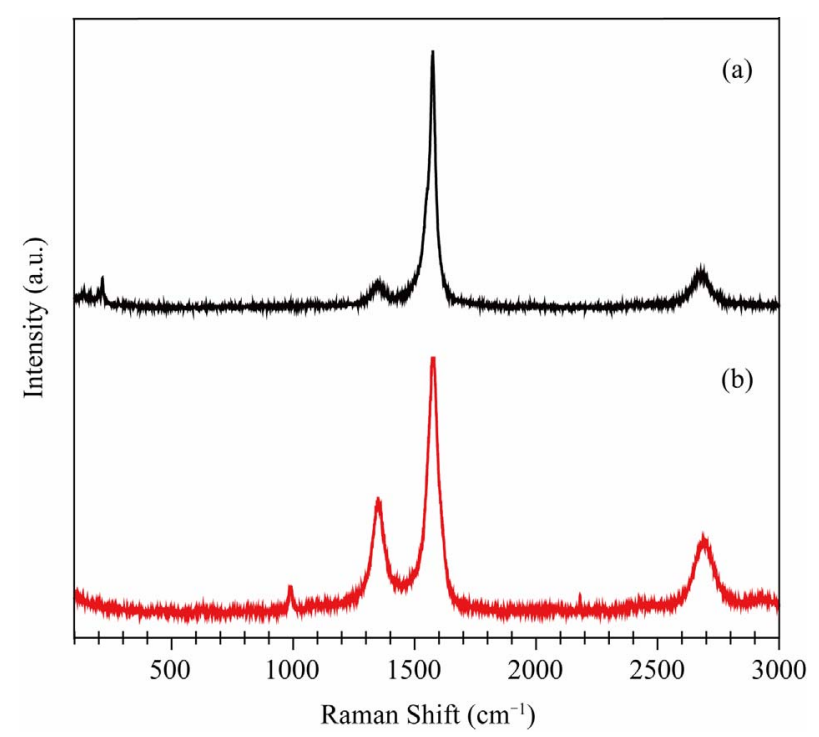

Figure 1. Raman spectra of SWNTs 3:1 $\mathrm{H}_{2} \mathrm{SO}_{4}: \mathrm{HNO}_{3}$ (a) pristine SWNT (b) refluxed for $6 \mathrm{~h}$. 
from the pristine sample as will be discussed subsequently.

Figure 2 shows the Raman spectra of the pristine and functionalized SWNTs; we have observed changes in relative intensity of the breathing modes in modified samples. These changes are indicator of chemical alteration of SWCNTs. It can be observed that the absolute intensities of the radial breathing mode are drastically reduced after functionalization. Rongmei et al. [15] found in $6 \mathrm{~h}$ sonication of SWNTs that high wave number RBM intensities decrease after which leads to assumption that destruction of small diameter SWCNT takes place $[13,16]$ or reduction in resonance enrichment of functionalized SWCNTs.

In our case, all low and high wave number RBM intensities has been decreased and disappeared. It may be due to long time acid treatment with 2 hours ultrasonication followed by $6-8$ hours acid refluxing. We believe that loss in the RBM intensities of SWNT is occurring due to the acid treatment, which breaks the translational symmetry of the SWNTs.

The D-band a useful diagnostic of disorder in the hexagonal framework of the tubes, whereas the relative intensity of the D- and G -bands is an important indicator of the amount of defects introduced upon chemical treatment [17]. The G-band, which is close to that for graphite, is related to the tangential (in-plane) mode of two carbon atoms in one graphene unit cell vibrating against one another, the so-called $E_{2 g}$ symmetry of the inner layer mode that represents the degree of crystallinity. This is a circumferential mode that vibrates perpendicularly to the tube axis. Figure 3 depicts the comparison of the intensity of D and $\mathrm{G}$ bands for the pristine and acid treated SWNTs. In comparison to the pristine tubes, after acid treatment, the intensity of the $\mathrm{sp}^{3} \mathrm{C}$ hybridization at $\sim 1348 \mathrm{~cm}^{-1}$ (D-band) has increased relative to the $\mathrm{sp}^{2} \mathrm{C}$ hybridization, in case of the acid treated sample due to the introduction of some functional groups on the side wall. This fact is verified by our data obtained from FTIR and UV-Vis-NIR spectroscopy. D-band peak (1348 $\mathrm{cm}^{-1}$ ) can be activated by disordering in the sidewall of the SWCNT and identified with sidewall defects. These defects disturb the aromatic system of electrons. G-band is the most intensive mode of SWCNTs which is observed at $\sim 1600 \mathrm{~cm}^{-1}$ and the atomic disarticulation is occurred along the circumferential direction in this mode [17]. $\mathrm{HNO}_{3}$ influences the most in the disorder band (D band), while $\mathrm{H}_{2} \mathrm{SO}_{4}$ is responsible for affecting graphite band ( $\mathrm{G}$ band) as well as the second order Raman band (G' band) [18]. The downshift in G-mode is interpreted as a softening and elongation of the $\mathrm{C}-\mathrm{C}$ bonds upon electron transfer to the SWCNTs, whereas electron withdrawal from the sp2 lattice leads to a contraction and

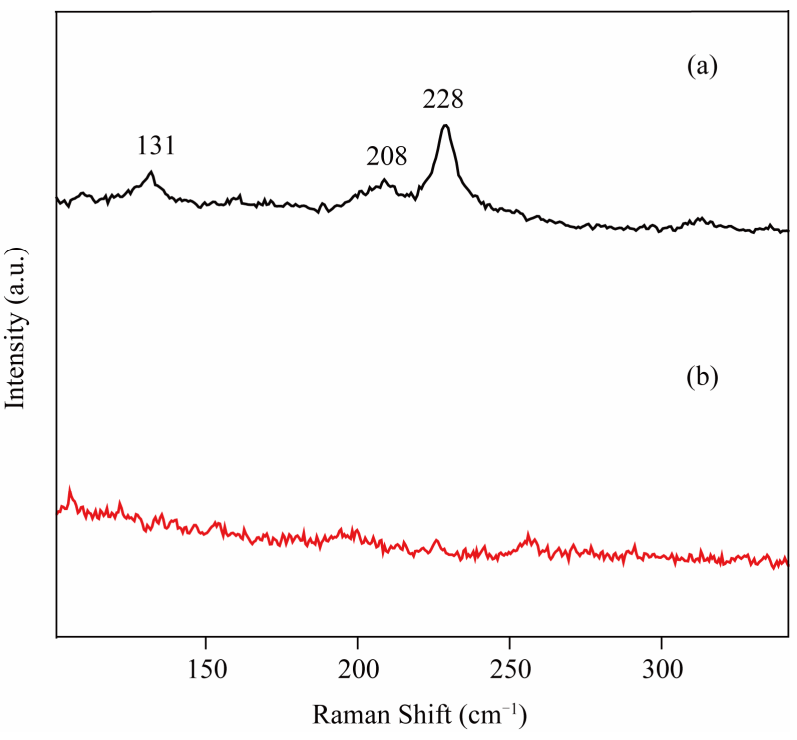

Figure 2. Raman Spectra of $\mathrm{RBM}$ mode of $3: 1 \mathrm{H}_{2} \mathrm{SO}_{4}$ : $\mathrm{HNO}_{3}$ (a) pristine SWNT (b) refluxed for $6 \mathrm{~h}$.

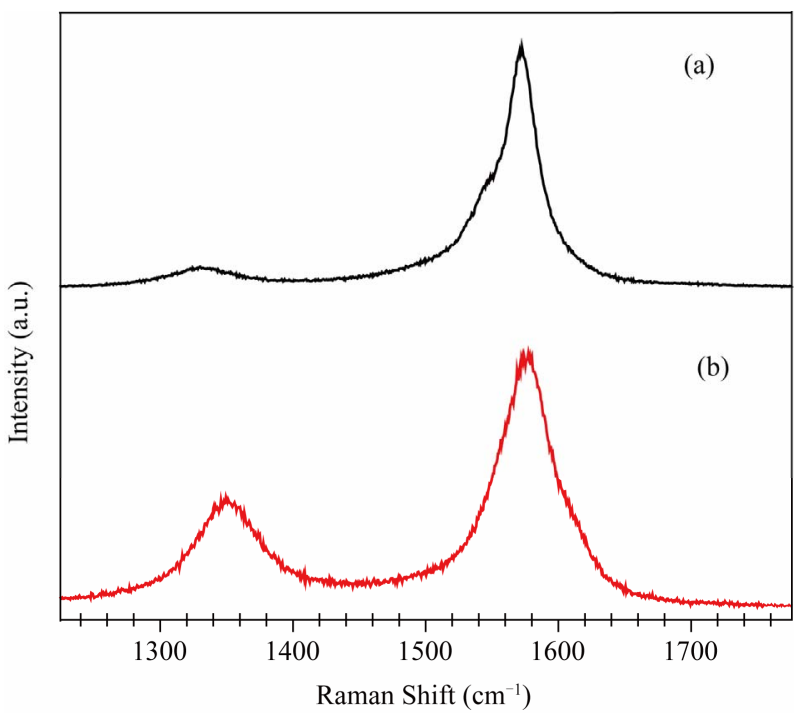

Figure 3. Raman Spectra of D- \& G- mode of 3:1 $\mathrm{H}_{2} \mathrm{SO}_{4}$ : $\mathrm{HNO}_{3}$ (a) pristine SWNT (b) refluxed for $6 \mathrm{~h}$.

hardening of the lattice. As nitric acid and sulfuric acid are known to lead to a $p$-type doping of SWCNTs, the observed upshifts of the tangential modes in acid-treated SWCNTs are very likely induced by intercalated acid molecules [13].Therefore, due to $\mathrm{HNO}_{3}$ and $\mathrm{H}_{2} \mathrm{SO}_{4}$ acids, there will be increase or decrease in the intensity of D-and G-band and also broadened G mode with a lower frequency. This broadening is related to the presence of free electrons in the metallic nanotubes and Breit-WignerFano (BWF) line is usually used to fit this G-mode broadening feature [11,17]. According to the Kataura-Plot, different photon energies determine whether the small diameter tubes that are resonantly enhanced are metallic 
or semiconducting [13]. At $\lambda_{\mathrm{exc}}=488 \mathrm{~nm}$ (Figure 3), the preferentially functionalized small diameter SWCNTs are semiconducting, therefore the G-band shows an increase in BWF asymmetry.

This BWF line is observed in n-doped graphite intercalation compounds (GIC), n-doped fullerenes, as well as metallic SWNTs [19]. The ratio of the D- to the G-band intensity i.e. $I_{d} / I_{g}$, is usually used for a measurement of the disordered sites on carbon nanotubes walls, and it is the indicator of the level of the covalent functionalization of the CNTs [14]. On comparing the modified and non modified tubes we have observed an increase in the ratio of intensities $R=I_{d} / I_{g}$ from 0.125 to 0.40 , for the modified tubes as is shown in Table 1.

Full width at half maximum (FWHM) of D- and G-peak was analyzed for pristine and functionalized sample of SWCNT (Table 1). For the G peak, the FWHM of the chemically treated SWCNT (i.e. $\Delta \omega_{G} \sim$ $46.0 \mathrm{~cm}^{-1}$ ) was much larger than that of the untreated samples (i.e., $\Delta \omega_{G} \sim 28.9 \mathrm{~cm}^{-1}$ ) and for $\mathrm{D}$ peak, the FWHM of the chemical treated SWCNT (i.e. $\Delta \omega_{D} \sim 47.4$ $\mathrm{cm}^{-1}$ ) was larger than that of the untreated samples (i.e., $\left.\Delta \omega_{D} \sim 31.7 \mathrm{~cm}^{-1}\right)$. This shows that increase in the FWHM of the D peak (i.e. $\Delta \omega_{D}$ ) is less than the $\mathrm{G}$ peak (i.e. $\left.\Delta \omega_{G}\right)$.

It is found that the ratio of the D- to G-mode intensity increase after functionalization [13]. Aryl-nanotube bond formed during the functionalization, which increases the intensity of D-peak and due to the electronic resonance, decreases the intensity of the tangential mode (G-peak). By these effects, increase in their peaks ratio $\left(I_{d} / I_{g}\right)$, indicates an increased disorder of the graphitic structure of the modified nanotubes [8], which shows that the nanotubes were covalently modified. Raman can detect changes in $\mathrm{C}-\mathrm{C}$ bond length. Due to electrochemical charge injection, the intensities of both the modes vary with the emptying/depleting or filling of the bonding and anti bonding states.

\subsection{FTIR Spectroscopy}

FTIR studies have been performed in the range 400 to $4000 / \mathrm{cm}^{-1}$ for the identification of the functional group attached on the surface of the CNTs. FTIR spectroscopy has been used extensively in the structural determination of molecules. Figure 4 shows a comparative FTIR data for the pristine and refluxed samples. As observed in the pristine sample there is almost no signal except for a small C-C stretch, however, on acid treatment quite a number of new peaks appear. The bands due to the $\mathrm{C}=\mathrm{O}$ stretch are very prominently seen in the range $1740 \mathrm{~cm}^{-1}$ for the carboxylated SWNT. The sample refluxed in $3: 1$ $\mathrm{H}_{2} \mathrm{SO}_{4}$ : $\mathrm{HNO} 3$ acid for $6-7 \mathrm{hrs}$ shows a distinct band at
Table 1. Comparison of the Raman spectral features of pristine and functionalized SWNT samples.

\begin{tabular}{cccc}
\hline \multirow{2}{*}{ Sample } & $\boldsymbol{I}_{d} / \boldsymbol{I}_{g}$ & \multicolumn{2}{c}{ FWHM $\left(\mathrm{cm}^{-\mathbf{1}}\right)$} \\
\cline { 3 - 4 } & & G band $\left(\Delta \omega_{G}\right)$ & D band $\left(\Delta \omega_{D}\right)$ \\
\hline Pristine & 0.125 & 28.9 & 31.7 \\
Functionalized & 0.40 & 46.0 & 47.4 \\
\hline
\end{tabular}

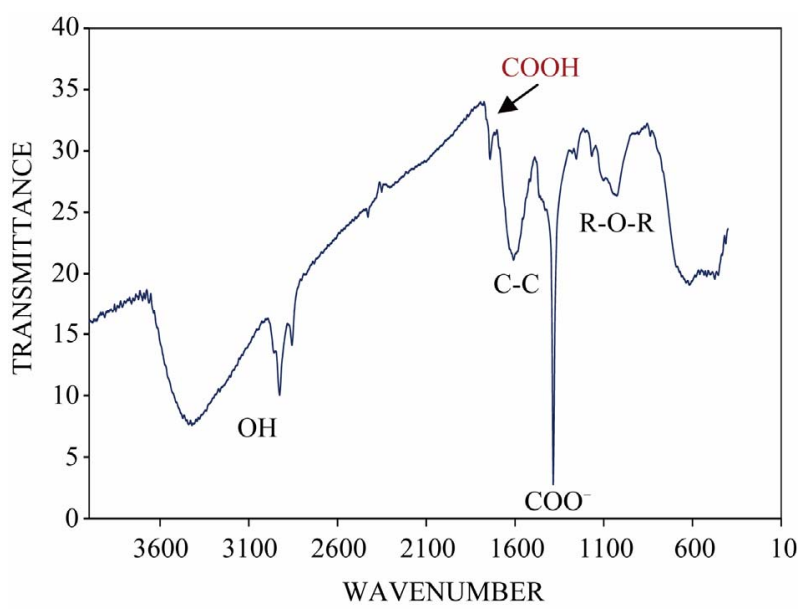

(a)

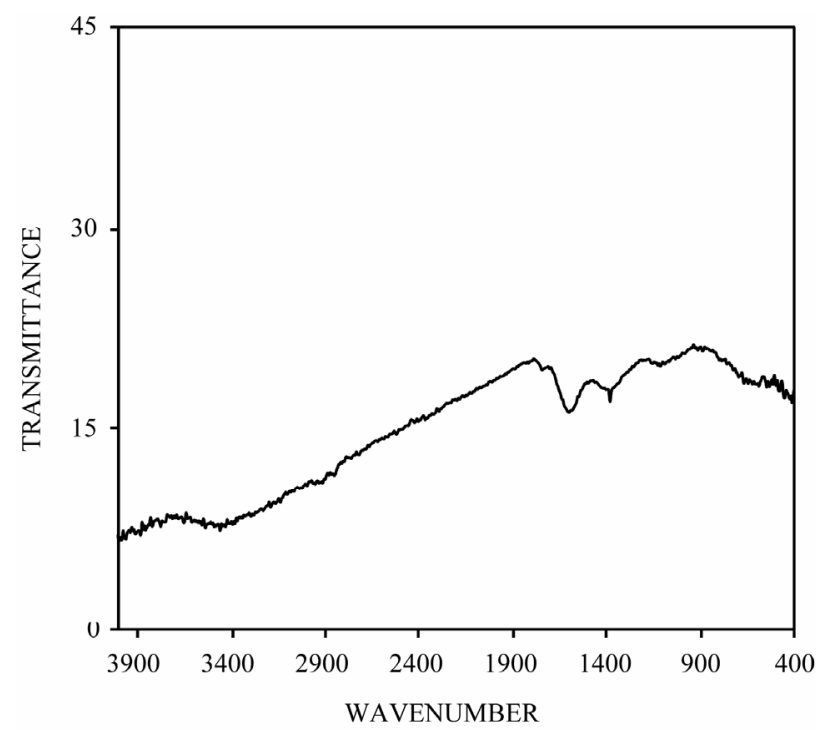

(b)

Figure 4. FTIR spectra of SWNTs 3:1 $\mathrm{H}_{2} \mathrm{SO}_{4}: \mathrm{HNO}_{3}$ (a) refluxed for $6 \mathrm{~h}(\mathrm{~b})$ pristine SWNTs.

$1740 \mathrm{~cm}^{-1}$, which can be assigned to the acid carbonyl-stretching mode (Figure 1(a)). Other bands seen in this sample are a small one at $2950 \mathrm{~cm}^{-1}$ and another at $3450 \mathrm{~cm}^{-1}$, that are characteristic of $\mathrm{C}-\mathrm{H}$ and $\mathrm{O}-\mathrm{H}$, stretches respectively. The $\mathrm{C}-\mathrm{C}$ vibrations occur due to the internal defects, and the $\mathrm{O}-\mathrm{H}$ vibration is associated 
with the amorphous carbon because amorphous carbon easily forms a bond with atmospheric air. However, the intensity of this O-H peak is relatively lower and shows that a lesser amount of amorphous carbon formed during growth [20].

Along with this an anti symmetric stretch C-O is also clearly distinguishable at $1659 \mathrm{~cm}^{-1}$. The bands at 1235 $\mathrm{cm}^{-1}$ and $1400 \mathrm{~cm}^{-1}$ are representative of $\mathrm{C}=\mathrm{C}$, this shift in band from $1600 \mathrm{~cm}^{-1}$ indicates the changes in the CNT structure upon carboxylation $[21,22]$.

\subsection{UV-VIS-NIR Spectroscopy}

Figure 5 depicts the absorption spectra of pristine SWNT and functionalized SWNTs. The pristine sample shows two distinct peaks at $960 \mathrm{~nm}$ and $750 \mathrm{~nm}$. These are the $\mathrm{S}_{11}$ and $\mathrm{S}_{22}$ transitions respectively, and they correspond to the first and second pair of van Hove singularities of the semiconducting nanotubes. The transitions corresponding to the metallic nanotubes are not observed.

Upon functionalization, the $\mathrm{S}_{22}$ peak vanishes. It is also seen that the intensity of the $S_{11}$ peak decreases as we move from the pristine sample to the refluxed sample. This implies that there is a change in the electronic structure of the CNTs on acid treatment. It has been reported $[22,23]$ that on covalent modification the CNTs results in a disruption of the aromatic system, which in turn causes a change in the conductivity. This gives rise to the changes observed in the van Hove singularities in the spectra. This is indicative of the fact that sidewall opening has taken place during acid treatment so as to accommodate the carboxyl group. An important consideration of this methodology is neutralization of the remaining acid. The sample degradation starts when exposed to prolonged acid exposure. Our experience with another sample validated the premise, that when kept overnight in the quenched state without neutralization the CNTs completely solubilise and pass through the filtration membrane without leaving a functionalized CNT residue upon it.

\section{Conclusions}

In our studies we have observed that acid treatment duration of only about $6-8$ hours is sufficient to functionalize the CNTs by introducing $\mathrm{COOH}$ group presumably at the CNT sidewalls. But to the best of our knowledge most literature cites a reaction duration ranging from 12 24 hours. In our studies, we have also observed that, refluxing duration of more than 10 hours completely damages the CNTs as observed by the intensity of the defect band in Raman spectra and complete disappearance of

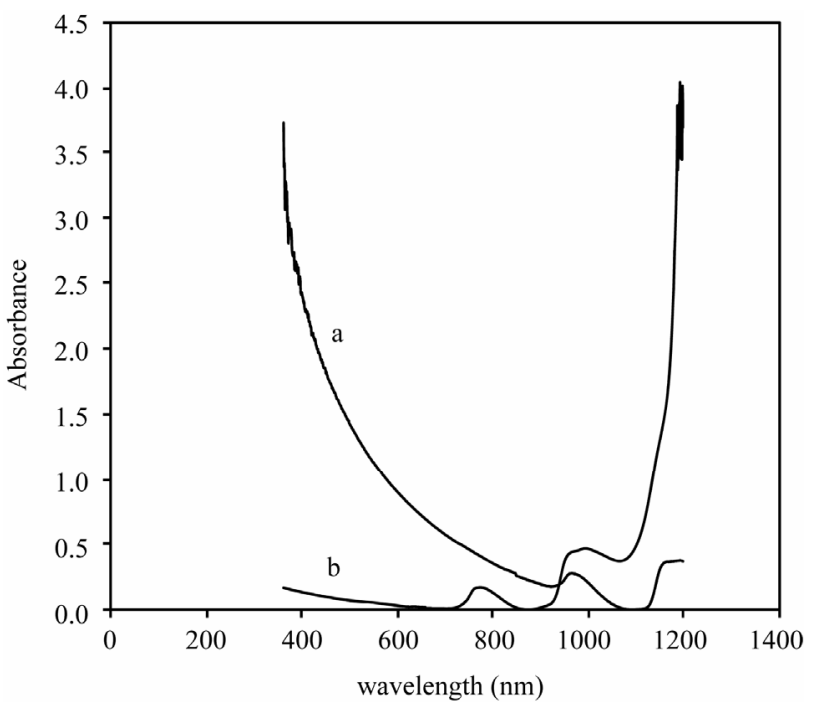

Figure 5. UV comparison of (a) $6 \mathrm{~h}$ refluxed sample, (b) and pristine SWNTs.

the characteristic $S_{11}$ and $S_{22}$ peaks of CNTs in the UV-Vis-NIR spectra.

\section{Acknowledgements}

The authors gratefully acknowledge the financial support provided by Ministry of Communication \& Information Technology, Govt. of India, through its Grant No. 20(14)/2007/NANO, dated. $20^{\text {th }}$ April 2010.

\section{References}

[1] E. T. Thostenson, Z. F. Ren and T. W. Chou, "Advances in the Science and Technology of Carbon Nanotubes and Their Composites: A Review," Composites Science and Technology, Vol. 61, No. 13, 2001, pp. 1899-1912. doi:10.1016/S0266-3538(01)00094-X

[2] S. Niyogi, M. A. Hamon, H. Hu, B. Zhao, P. Bhowmik, R. Sen, M.E.Itkis and R. C. Haddon, "Chemistry of SingleWalled Carbon Nanotubes," Accouts of Chemical Research, Vol. 35 No. 12, 2002, pp. 1105-1113. doi:10.1021/ar010155r

[3] L. A.Girifalco, M. Hodack and R. S Lee, "Carbon Nanotubes, Buckyballs, Ropes, and a Universal Graphitic Potential," Physical Review B, Vol. 62, 2000, pp. 13104 13110. doi:10.1103/PhysRevB.62.13104

[4] D. J. Nelson, H. Rhoads and C. Brammer, "Covalently Sidewall-Functionalized SWNTs," Journal of Physical Chemistry C, Vol. 111, No. 48, 2007, pp. 17872-17878. doi:10.1021/jp071326y

[5] G. W. Lee, J. Kim, J. Yoon, J. S. Bae, B. Shin, I. Kim, W. Oh and M. Ree, "Structural Characterization of Carboxylated Multi-walled Carbon Nanotubes," Thin Solid Films, Vol. 516, No. 17, 2008, pp. 5781-5784. doi:10.1016/j.tsf.2007.10.071 
[6] C. H. Lau, R. Cervini, S. R. Clarke, M. G. Markovic, J. G. Matisons, S. C. Hawkins, C. P. Huynh, and G. P. Simon, "The Effect of Functionalization on Structure and Electrical Conductivity of Multi-walled Carbon Nanotubes," Journal of Nanoparticle Research, Vol. 10, 2008, pp. 7788. doi:10.1007/s11051-008-9376-1

[7] M. S. D. Lekgoathi, J. Heveling, W. G. Augustyn, S. J. Husselman, P. G. Masha and S. Rossouw, "Effect of Carboxylate Functional Groups on the Surface Area of SWCNTs," International Journal of Nanotechnology and Applications, Vol. 2, No. 2, 2008, pp. 141-148.

[8] S. Costa, B. Scheibe, M. Rummeli, and E. B. Palen, "Raman Spectroscopy Study on Concentrated Acid Treated Carbon Nanotubes," Physica Status Solidi (b), Vol. 246, No.11-12, 2009, pp. 2717-2720. doi:10.1002/pssb.200982297

[9] G. Ovejero, J. L. Sotelo, M. D. Romero, A. Rodriguez, M. A. Ocana, G. Rodriguez and J. Garcia, "Multiwalled Carbon Nanotubes for Liquid-Phase Oxidation. Functionalization, Characterization, and Catalytic Activity," Industrial \& Engineering Chemistry Research, Vol. 45, No. 7, 2006, pp. 2206-2212. doi:10.1021/ie051079p

[10] V. Skakalova, A. B. Kaiser, U. D. Weglikowska, K. Hrncarikova and S. Roth, "Effect of Chemical treatment on Electrical Conductivity, Infrared Absorption, and Raman Spectra of Single-Walled Carbon Nanotubes," Journal of Physical Chemistry B, Vol. 109, No. 15, 2005, pp. 7174-7181. doi:10.1021/jp044741o

[11] S. C. Tsang, P. J. F.Harris, and M. L. H.Green, "Thinning and Opening of Carbon Nanotubes by Oxidation using Carbon Dioxide," Nature, Vol. 362, 1993, pp. 520-522. doi: $10.1038 / 362520 \mathrm{a} 0$

[12] H. T. Gomes, P. V. Samant, P. Serp, P. Kalck, J. L. Figueiredo and J. L. Faria, "Carbon Nanotubes and Xerogels as Supports of Well-Dispersed Pt Catalysts for Environmental Applications," Applied Catalysis B: Environmental, Vol. 54, No. 3, 2004, pp. 175-182. doi:10.1016/j.apcatb.2004.06.009

[13] R. Graupner, "Raman Spectroscopy of Covalently Functionalized Single-wall Carbon Nanotubes," Journal of Raman Spectroscopy, Vol. 38, 2007, pp. 673-683. doi:10.1002/jrs.1694

[14] S. Mirershadi, S. Z. Mortazavi, A. Reyhani, N. Moniri and A. J. Novinrooz, "Effective Condition for Purification of Multi-Walled Carbon Nanotubes by Nitric Acid," Synthesis and Reactivity in Inorganic, Metal-Organic and Nano-Metal Chemistry, Vol. 39, No. 4, May 2009, pp. 204-208.
[15] R. Liu, "The Functionalization of Carbon Nanotubes," Ph.D. Dissertation, School of Chemistry, University of NS W, Sydney, 2008.

[16] K. H. An, J. S. Park, C.M. Yang, S. Y. Jeong, S. C. Lim, C. Kang, J. H. Son, M. S. Jeong and Y. H. Lee, "A Diameter-Selective Attack of Metallic Carbon Nanotubes by Nitronium Ions," Journal of American Chemical Society, Vol. 127, No. 14, 2005, pp. 5196-5203. doi:10.1021/ja0428199

[17] M. Burghard, "Electronic and Vibrational Properties of Chemically Modified Single Wall Carbon Nanotubes," Surface Science Reports, Vol. 58, No. 1-4, 2005, pp. 1109.

[18] A. G. Osorio, I. C. L Silveira, V. L. Bueno and C. P. Bergmann, "H2SO4/HNO3/HCl-Functionalization and Its Effect on Dispersion of Carbon Nanotubes in Aqueous Media," Applied Surface Science, Vol. 255, No. 5, 2008, pp. 2485-2489. doi:10.1016/j.apsusc.2008.07.144

[19] A. Jorio, M. A. Pimenta, A. G. Souza Filho, R. Saito, G. Dresselhaus and M. S. Dresselhaus, "Characterizing Carbon Nanotube Samples with Resonance Raman Scattering," New Journal of Physics, Vol. 5, No. 1, 2003, p. 139. doi:10.1088/1367-2630/5/1/139

[20] E. Titus, N. Ali, G. Cabral, J. Gracio, P. Ramesh Babu, and M. J. Jackson ,"Chemically Functionalized Carbon Nanotubes and Their Characterization Using Thermogravimetric Analysis, Fourier Transform Infrared, and Raman Spectroscopy," Journal of Materials Engineering and Performance, Vol. 15, No. 2, 2006, pp. 182-186. doi:10.1361/105994906X95841

[21] C. A. Dyke and J. M. Tour, "Covalent Functionalization of Single-Walled Carbon Nanotubes for Materials Applications," Journal of Physical Chemistry, Vol. 108, No. 51, November 2004, pp. 11151-11159.

[22] J. L. Bahr, J. Yang, D. V. Kosynkin, M. J. Bronikowski, R. Smalley and J.M. Tour, "Functionalization of Carbon Nanotubes by Electrochemical Reduction of Aryl Diazonium Salts: A Bucky Paper Electrode," Journal of American Chemical Society, Vol. 123, No. 27, 2001, pp. 6536-6542. doi:10.1021/ja010462s

[23] V. A. Sinani, M. K. Gheith, A. A. Yaroslavov, A. A. Rakhnyanskaya, K. Sun, A. A. Mamedov, J. P Wicksted and N. A. Kotov, "Aqueous Dispersions of Single-wall and Multiwall Carbon Nanotubes with Designed Amphiphilic Polycations," Journal of American Chemical Society, Vol. 127, No. 10, 2005, pp. 3463-3472. doi:10.1021/ja045670+ 